\title{
Feasible Scenario Spaces: a new way of measuring capability impacts
}

\author{
Fred DJ Bowden, Brandon Pincombe and Peter B Williams \\ Joint and Operations Analysis Division, Defence Science and Technology Group \\ Email: fred.bowden@defence.gov.au
}

\begin{abstract}
The future is unknowable, but organisations can still prepare for it. Traditional scenario methods do this by constructing a set of scenarios representing the critical uncertainties associated with the future. Scenarios are points or small regions within a large multi-dimensional space of possible futures. Options are tested against these scenarios to determine how they perform. For example, the Australian Department of Defence tests proposed capability sets using the Australian Capability Context Scenarios (ACCS), representing hypothesised critical uncertainties about future operations.
\end{abstract}

The ACCS represent only a small part of the space of all possible futures and any assessment against them provides only a limited view of how a capability will perform in the future. Rather than evaluating options against proposed scenarios we propose asking "what parts of the scenario space does a given capability set allow you to achieve with acceptable risk?" defined as the Feasible Scenario Space (FSS).

Unfortunately, as you move further into the future, the scenario space gets larger as there is more uncertainty of what the future might look like. This makes defining the FSS through exploration of the scenario space much more resource intensive. To make the assessment of options tractable we propose focusing on the scenarios (the key approved futures), the ACCS in the case of Defence, as the seed locations to start this search. The options are then compared based on the size of the hyper-volume surrounding the scenarios in which a given capability set presents an acceptable risk. The edges of this hyper-volume are estimated by testing options against points along each of the critical uncertainties moving out from each given seed scenario until a breaking point is found.

The FSS is defined as a surface which covers the set of scenario parameters for which a given capability set can achieve success, within acceptable levels of inherent risk.

This paper provides a detailed definition of the FSS and explains how to describe it within a multidimensional scenario space and how to test for the risk boundaries. An illustrative example is provided to show of how this approach would be applied.

Keywords: Force design, scenario analysis, modernisation 


\section{INTRODUCTION}

The future is unknowable, but organisations can still prepare for it. How you prepare for the future depends upon how constrained your boundaries are and how complex you expect the future will be within those bounds. Deterministic solutions work for simple and well constrained problems. Slightly more complex problems sit in the world of stochastic risk but, unfortunately, tractable solutions typically require ergodicity (i.e. stationarity - where the mean and variance of the stochastic process does not change over time -- and regularity - where any subsample of the stochastic process represents the average statistical properties of the entire process). In the absence of ergodicity - and, in truth, future security threats are inherently non-ergodic - we need to deal with irreducible uncertainty (Scholz and Reid, in publication). For any finite body of evidence there is an infinite number of generating functions that could have produced exactly that set of evidence and, even if you can discern what some of those generating functions are, which frequently you can't, they will differ from each other in what they predict future events to be and you can't know which is the right generating function (Scholz and Reid, in publication). One answer to this problem is to only ever hold provisional ideas and to actively look for dis-confirmatory evidence (Scholz and Reid, in publication). This is why, for military planners, history provides a useful but imperfect source of dis-confirmatory evidence.

Groups as diverse as climate scientists (Morgan et al., 2009) and oil field developers (Begg et al., 2014) have struggled with unknowable futures whether they have called this deep uncertainty, irreducible uncertainty, model uncertainty, Knightian uncertainty or ambiguity. Their responses have been to develop preparatory scenarios, without probabilities associated with them, covering identified critical uncertainties; develop the set of critical uncertainties through expert elicitation; where possible turn long term decisions into a series of short term ones; and implement the most plastic options, ones most likely to be able to morph or evolve to cope with whatever actually does occur in the future (Morgan and Mellon, 2011, Begg et al., 2014, Scholz and Reid, in publication). This ascertainment of the critical uncertainties, sometimes referred to as dimensions, of the scenario space takes methodological sophistication (Pincombe et al., 2013) because of the requirement to poll a representative sample of experts (Parente and Anderson-Parente, 2011 and Bolger and Wright 1994) who have learnt judgment in the problem space (Bolger and Wright 1994) and are motivated to contribute (Parente and Anderson-Parente, 2011) to reduce biasing effects (Koehler, Brenner, \& Griffin, 2002). These dimensions have been used to assess options against one super complex scenario (Pincombe and Pincombe, 2010), a small number of representatively diverse scenarios (Wright and Cairns, 2011) or millions of computational scenarios (Baker et al., 2007). All of these approaches discretize the scenario space and assess options against scenarios. This is turned around and it is propose that the comparison of options be based on the size of the hyper-volume within the scenario space to which each option provides access to - the Feasible Scenario Space (FSS). This idea is similar to that defined by Davis (Davis, 2012), however, whereas Davis expressed an "intent ... to examine as much of the possibility space ... as possible" the FSS concentrates on ascertaining the boundary of the feasible area; this focus makes the problem much more computationally tractable. In addition to this the FSS is identified within a scenario space defined by dimensions specifically tailored to future land warfare problems (Pincombe et al., 2013) whereas Davis (2012) only made the general suggestion of using some dimensions of uncertainty.

\section{DEFINING THE FEASIBLE SCENARIO SPACE}

The possible future space can be considered as a multi-dimensional space with dimensions defined by Own Force Capabilities, Physical Environment, Human Terrain, Operational Partnerships, Socio-political Issues and Nature, Diversity and Intensity of Threat (Pincombe et al., 2013). Generally one of these dimensions will be the focus of the study, for example Own Force Capabilities if you are considering changes to your force, so the remaining five dimensional space represents the scenario space in which we could consider, say, a set of Own Force Capability options. In this case, the FSS for a given option is defined as those parts of the Scenario Space within which each is able to operate.

To illustrate the FSS, let us first consider one dimension, say Physical Environment, and one option, say a wheeled combat vehicle capability, or a sub-surface maritime vehicle capability, or a flying autonomous vehicle capability. The boundary of the FSS would be defined as those physical environments in which the tested capability is able to operate with acceptable risk. Obviously the wheeled vehicle cannot fly, if it can swim it will only swim at low sea states, and certain ground pressures, slopes and vegetation may deny access or canalise movement; the submarine will be unable to fly or drive but will also have a maximum operating depth; the flying system will have height and wind restrictions. These vehicles may be able to operate at higher sea states, shallower depths, or greater wind speeds; but not at acceptable risk. The 
operational boundary, produced from the limits of the option, on the dimension form's the FSS in this one dimensional case.

The FSS becomes more complicated as more dimensions are considered - now there is the need to consider how variations in combinations of dimensions might change the level of is acceptable risk. For example, consider how the dimensions of Nature, Diversity and Intensity of Threat and Physical Environment interact

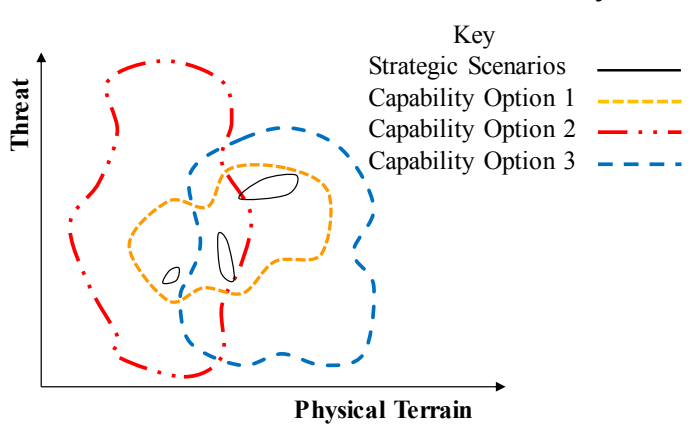

Figure 1. Notional Feasible Friendly Scenario Space. within the context of a combat vehicle. Continuing the earlier example, consider the option of a wheeled combat vehicle within a jungle physical environment with a mine or improvised explosive device threat. In this case there would be unacceptable risk as the canalising effect of the jungle makes it very easy for the enemy to predict where the vehicle is likely to operate and thus place mines in those location. This was a lesson learnt by the Indian Army when operating in Sri Lanka (Nadkarni, 2006).

The concept of the FSS in two dimensions is illustrated in Figure 1. In the figure the areas enclosed in black lines represent those areas of the state space covered by strategic guidance such as the ACCS (Department of Defence, 2010), the Canadian Arctic Planning Scenarios (Mugridge, Avis \& Race, 2011) or any of their many equivalents. This has been represented by three distinct areas, representing three possible future scenarios. Depending on strategic guidance, this may actually be a single point in the scenario space or more than three areas.

The yellow, red and blue areas represent the areas of the scenario space that can be covered by three different possible force options. The red and blue options cover large regions of the future scenario space but neither of them completely covers all of the area of the space specified by strategic guidance (the black boundaries). Only the yellow option covers the areas of strategic guidance. However, it covers much less area than the other two options. What is gained and lost by all three options is clear. By representing the possible outcomes in this way it is clear which parts of the scenario space each option is able to operate allowing a comparison of how much of the possible future each option will be able to operate in.

To this point the discussion has been based around the effect of a given capability on what the Friendly force is able to do, this is defined as the Feasible Friendly Scenario Space (FFSS). Differences in FFSS define how different options enhance or diminish the capability of the force being studied. So FFSS defines those areas of the future scenario space in which friendly forces are able to operate in with acceptable risk.

Generally capabilities not only impact friendly force capabilities but also restrict what the enemy is able, or willing, to do. For example, having superior reconnaissance and surveillance prevents the enemy from conducting operations in open battle formations, forcing operations into the complex physical and human terrain. So, the principle impact of a capability may be to restrict the freedom of action of the enemy. Just as with the FFSS this area defines the boundary of the scenario space in which the enemy can operate with acceptable risk. This is defined as the feasible enemy scenario space (FESS).

It is the combination of the differences in both the FFSS and FESS that determine the overall impact of a given change in the future force. From now on in this paper the FSS refers to both the FFSS and FESS unless they are explicitly mentioned.

In essence, the FSS is defined as a surface which covers the set of scenario parameters for which a given capability set can achieve success, within acceptable levels of inherent risk.

Even though the FSS defines a region of the study space within which a particular variable set is successful, the most important information lies at the boundary of the surface. This boundary defines the set of break points in the study; the reasoning that defines the fulcrum between success and failure, or acceptable and unacceptable levels of risk, is often much more informative than knowing how much of the space is covered. It is therefore the analyst's primary objective to identify both when a capability transitions from success to failure (or vice-versa) and why. This understanding can then be used to extrapolate the FSS in cases where exhaustive data cannot be generated.

The exact method used for mapping the FSS boundary will depend on the nature of the problem, the nature of the experiment and the relevant dimensions. For example, a tightly-constrained problem with readily 
enumerable variables could be modelled in a closed-loop simulation. In this instance, it is possible to run parametric analyses across all permutations of the dimensions to generate the FSS exhaustively. A crisp boundary can be defined, which can be based on calculated, objective metrics (such as number of casualties, time to complete mission, or percentage of enemy craft detected). It is also possible to investigate the complex interactions from cross-variable non-linearities. The downside, however, is that without further investigation, the why behind dimension interactions and the FSS boundary itself is missing, and relies on the analyst to infer reasoning from the when.

To serve as a counterpoint, a coarse seminar wargame will take the reverse approach: seeking to understand the why in order to map the when. That is, it becomes the role of the analyst to delve into all the possible conditions (or combinations of dimensions) that will contribute to forming the shape of the FSS. They need to find the underlying causes (either from a single dependent variable, or from multiple simultaneous changes) to find the break points of the system. The restriction of this method is that the exact boundary of any FSS is by nature an approximation, or fuzzy, and it becomes difficult and open to interpretation to compare several feasible spaces against each other. At best, a risk assessment can be used as the metric delineating success (an acceptable level of risk) and failure (an unacceptable level of risk). In this case, the frequency-consequence distribution forms a fuzzy boundary for the FSS [Bilusich et al. (2015)].

\section{UNDERSTANDING THE PROBLEM SPACE: DIMENSION CLASSES AND SUCCESS}

In order to concisely map the problem space graphically (as in Figure 1), the analyst needs to be aware of how the dimensions behave, and how to define the boundary of the FSS itself.

The first thing that needs to be defined when considering this approach is which dimensions are relevant to the particular study being conducted. Pincombe et al. (2013) define six dimensions but not all of these are relevant in all studies. For example, in considering a new fighter capability the key dimensions that need to be considered are the Physical Environment and the Nature, Diversity and Intensity of Threat. In most major system-based capabilities the focus is on the dimensions of Physical Environment, Human Terrain, and Nature, Diversity and Intensity of Threat with Own Force Capabilities being the primary independent variable.

The next step is to classify the dimensions into sub-classes: parameters and variables.

Essentially, we define a study parameter to be any nominal dimension. That is, they have no inherent value, ranking or logical ordering. The study variables can then be either ordinal, ratio or interval data; it can be objectively determined if one value of that dimension is 'better' than another. Table 1 lists some possible examples across the land, sea and air domains.

Table 1. Dimension Classes with examples for different domains.

\begin{tabular}{|l|l|l|l|}
\cline { 2 - 4 } \multicolumn{1}{c|}{ Land } & \multicolumn{1}{c|}{ Sea } & \multicolumn{1}{c|}{ Air } \\
\hline Parameters & Environment Type & Permissive Environment & \\
& $\begin{array}{l}\text { Mission Type } \\
\text { Support from local govt }\end{array}$ & $\begin{array}{l}\text { Mission Type } \\
\text { ROE }\end{array}$ & Mission Type \\
& TTPs \\
\hline & $\begin{array}{l}\text { Future Capability Types } \\
\text { Enemy Strength } \\
\text { Resistance from local } \\
\text { population }\end{array}$ & $\begin{array}{l}\text { Sea State } \\
\text { Number of Enemy }\end{array}$ & $\begin{array}{l}\text { Airframe Type } \\
\text { Number of Enemy }\end{array}$ \\
\hline
\end{tabular}

In practice, the variables appear as axes on the figure - with the prime dependent variable, the main focus of the study, as the horizontal axis. Then a separate figure is produced for each of the parameters. The FSS is the amalgamation of all information; however, it does not make sense to reduce it to a single picture.

\section{INTERPRETING AND USING THE FEASIBLE SCENARIO SPACE}

Feasible Scenario Spaces are powerful tools for explaining the complex interactions of capabilities, concepts and structures within uncertain futures. However, care needs to be taken in their interpretation and for underpinning key decisions. If the FSS for one option dominates another, then the solution is trivial, else the application of the FSS concept becomes complex very quickly.

The why at each edge of the boundary should give insight into how the FSS could be expanded for an option; which of the dimensions will yield the greatest increase for the least cost? Additionally, the analyst or client 
can place weightings on which regions of the scenario space are most important, based on strategic guidance for example. One thing that should be considered when trading-off different options is the contribution to the FSS from the FFSS and the FESS respectively; one option could provide a benefit in the Friendly space, whilst another could provide a benefit in the FESS space.

\section{APPLICATION TO JOINT OFFENSIVE SUPPORT}

Consideration of joint offensive support (JOS) will be used to demonstrate the application of the FSS approach. What is presented here is a greatly redacted example, and so the results here only illustrate the FSS method and should not be considered fully representative of an extant analysis. For example, it does not consider the different types of offensive support (OS) missions that might need to be conducted. Within this construct the following JOS assets are considered: close air support (CAS), armed reconnaissance helicopters $(\mathrm{ARH})$, naval gunfire support (NGS), offensive fires from towed 155-mm artillery (OF), 81-mm mortars (M) and a (conjectural) ship-based loitering munition (LM).

For brevity, only three dimensions of the scenario space will be considered: physical environment, human terrain and threat. In each dimension a reduced number of components are analysed. For the physical environment dimension two components are used: distance from the coast (to determine whether NGS can target, or if other systems can manoeuvre within range in a reasonable time) and sea states (to determine whether it is safe for NGS to fire or for ARH to take off). For the human terrain dimension a single component is applied: distance to civilians/sensitive sites (this is measured based on the safety distance of the asset and the consequences of impacting these sites). The threat dimension utilises two components: counter battery fires (CBF) (whether a particular system is susceptible to counter battery attack) and time covered (the duration for which a system can provide sustained fires).

This paper uses notional assessments of the six systems against the components of the dimensions as shown in Table 2. In this table the data has been normalised so that 1 represents being able to completely address the scenario component and 0 is complete failure. In should be noted in reality that this assessment would have many more aspects to it and it is likely that it will be impossible to represent each system in relation to a single component by a single number.

Table 2. Fictional assessment of JOS assets against dimension components.

\begin{tabular}{|c|cc|c|cc|}
\cline { 2 - 6 } \multicolumn{1}{c|}{} & \multicolumn{2}{c|}{ Physical } & Human & \multicolumn{2}{c|}{ Threat } \\
\cline { 2 - 6 } \multicolumn{1}{c|}{} & Distance from Coast & Sea State & Safety Distance & CBF & Time Covered \\
\hline CAS & 1 & 1 & 0.1 & 1 & 0.25 \\
ARH & 0.8 & 0.5 & 0.94 & 1 & 0.4 \\
NGS & 0.1 & 0.75 & 0.3 & 0.8 & 1 \\
OF & 0.7 & 1 & 0.5 & 0.2 & 1 \\
M & 0.9 & 1 & 0.8 & 0 & 0.5 \\
LM & 0.3 & 0.75 & 0.4 & 1 & 0.25 \\
\hline
\end{tabular}

As part of the analysis three options are considered, these are given in Table 3. The first is based around the idea that all OS is provided by Joint Force. The second option considers only two land-based systems being

Table 3. JOS options.

\begin{tabular}{|l|l|l|l|}
\hline System & Option 1 & Option 2 & Option 3 \\
\hline CAS & $\checkmark$ & & \\
\hline ARH & & $\checkmark$ & \\
\hline NGS & $\checkmark$ & & \\
\hline OF & & $\checkmark$ & \\
\hline M & & & $\checkmark$ \\
\hline LM & $\checkmark$ & & $\checkmark$ \\
\hline
\end{tabular}
available. The final case is a pairing based on a minimalist approach of mortars and loitering munitions.

First, consider the case where a future option is chosen through scenario analysis conducted around a future consistent with the Dibb Report of 1986, in which "air and naval forces ... [deny] the sea and air gap ... preventing any successful landing of significant forces on Australian soil" but "lesser enemy forces might land [and] we will need ... to protect our military installations, infrastructure and civilian population in the north" (Dibb, 1986, p. 5). Thus, enemy special forces operate in coastal northern Australia, mostly within $50 \mathrm{~km}$ of the coast at sea level, during the dry season with low sea states $(\leq 2)$, in sparsely populated areas with little need to consider safety ranges (i.e. $500+\mathrm{m}$ ) and minimal counter battery fire provided by mortars. Within the context of this scenario the 
distance from the coast and safety distance and system susceptibility to CBF do not distinguish between the JOS assets or options. So based on a traditional scenario-based analysis there are only two components that need to be considered in the analysis: sea state and time covered. All three options presented in Table 3 are able to meet the needs of this scenario. Given all three options can achieve the effects required for the scenario the next step would be to look at which option best meets the two distinguishing components. The data shows that Option 1 exceeds the requirements four times, Option 2 two times and Option 3 three times. Assuming both components are equally important Option 1 best meets the needs to the scenario.

Alternatively, taking the key scenario dimensions as the physical, human and threat environments a representation of the $\mathrm{FFSS}^{1}$ is given in Figure 2. This representation is in the form of spider diagrams where each axis is a component of the scenario space and the value of a given option is that provided by taking the maximum of the assessment of the component systems ${ }^{2}$. In this figure the shaded area provides a representation of the area of the scenario space as covered by the option and the blue line represents the requirements as specified by the Dibb related scenario. Clearly based on the FFSS the Option 2 covers more of the scenario space than covered by the other two options. So based on a the FSS method in preparing for an irreducibly uncertain future to ensure an ability to operate in more possible futures Option 2 is the logical choice.
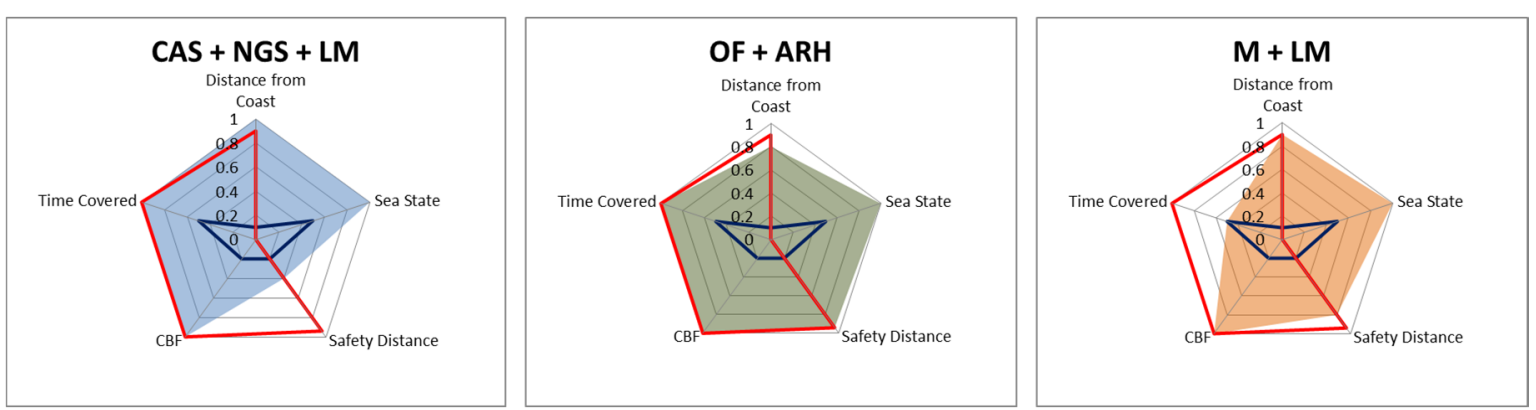

Figure 2. Example Joint Offensive Support Feasible Friendly Scenario Spaces.

Consider a scenario when the future is not as described early but closer to that of a putative conflict in the Middle East Area of Operations (MEAO) where the situation is $\geq 500 \mathrm{~km}$ inland (thus the sea state is unimportant) and the enemy operates amongst civilians (making a $50 \mathrm{~m}$ safety range desirable). This enemy can undertake counter battery fire and we have a need to keep them covered with JOS for extended durations of time. The requirements of this scenario are represented within the figure by the red line. In this case Option 2 is the clear winner. This shows the fragility of conducting analysis against individual scenarios and the value of considering FSS as a whole. It allows the decision-maker to better understand what is able to be covered by the options being considered, and not simply be aware of the effectiveness of each option within a given set of possible future scenarios. It could be argued that the use of multiple scenarios overcomes this issue. However, this simply changes the number of scenarios each option is considered against, and defining the FSS clearly shows the decision-maker what each option is able to do with acceptable risk and what parts of the future scenario space an option is unable to operate within.

As discussed earlier, defining the FSS also allows you to better understand how you might expand a given option in a particular direction. For the MEAO scenario above, none of the options are able to meet all the requirements. The closest is that of Option 2, which falls short on its ability of operate long distances from the coast. So the question might be asked how can this be improved? Alternatively, it might be easier to improve the safety distance ability of Option 1. By understanding the boundaries of acceptable risk of different options it is possible to better explore how to extend these boundaries and where shortfalls might occur in the future.

\section{CONCLUSION}

This paper has presented the idea of the FSS as a new way of evaluating options and their ability to perform in the future. Rather than providing a decision-maker with the performance of options within a small set of possible futures it asks within what possible futures can a given option effectively operate within. Although the approach is more difficult it also provides a richer understanding of the impact of a given option across the variety of possibly futures that an organisation might face. A JOS example is used to show how the

\footnotetext{
${ }^{1}$ In this example only the FFSS is considered. However, a similar analysis could be conducted to determine the impact of these two options on the FESS.

${ }^{2}$ Once more it is noted that this assessment is very simplistic and has been used for illustrative purposes only.
} 
Bowden et al., Feasible Scenario Spaces: a new way of measuring capability impacts

method can be used in practice. This also shows that the method is applicable not only to assessment of future capabilities but also to the examination of the flexibility of force structures to deal with the unknown of today.

\section{ACKNOWLEDGMENTS}

The authors acknowledge Dean Bowley, Anne-Marie Grisogono, Don Lowe and Darryn Reid for their contributions to the early thinking in this space.

\section{REFERENCES}

Baker, S, Bender, A, Abbass, H and Sarker, R (2007), A Scenario-based Evolutionary Scheduling Approach for Assessing Future Supply Chain Fleet Capabilities. In K Dahal, KC Tan and PI Cowling (Eds.), Evolutionary Scheduling (pp. 485-511). Berlin: Springer.

Begg, S., Bratvold, R., \& Welsh, M. (2014), Uncertainty vs Variability: what's the difference and why is it important?, Paper presented at the SPE Hydrocarbon Economics and Evaluation Symposium, Houston.

Bilusich D, Lord S \& Nunez-Vaz R (2015), The implications of empirical data for risk, Journal of Risk Research, 18:4, 521-538, DOI: 10.1080/13669877.2014.910682.

Bolger, F and Wright, G (1994), Assessing the quality of expert judgement, Decision Support Systems, 11, 124.

Bowden, FDJ, Finlay, L, Lohmeyer, D, and Stanford, C, Multi-Method Approach to Future Army SubConcept Analysis, 18th World IMACS Congress and MODSIM09 International Congress on Modelling and Simulation, Cairns, Australia, 13-17th July 2009.

Davis, P (2012). Lessons from RAND's work on planning under uncertainty for national security. RAND Corporation, TR-1249, Santa Monica, Calif.

Department of Defence (2010). The Strategy Framework 2010. Department of Defence, Canberra, ACT.

Dibb, P. (1986). Review of Australia's Defence Capabilities. Australian Government Publishing Service, Canberra.

Morgan, MG, Dowlatadabi, H, Henrion, M, Keith, D, Lempert, R, McBride, S, Small, M and Wilbanks, T (2009), Best Practice Approaches for Characterizing, Communicating and Incorporating Scientific Uncertainty in Climate Decision Making. Synthesis and Assessment Product 5.2. National Technical Information Service.

Morgan, MG and Mellon, C, (2011), Certainty, uncertainty, and climate change. Climatic Change, 108(4), 707-721.

Mugridge, D., Avis, P. and Race, P. (2011). Arctic Planning Scenarios. DRDC CORA CR 2011-117.

Nadkarni, R (MAJGEN ret. Indian Army) (2006), Personal correspondence.

Parente, R and Anderson-Parente, J (2011), A case study of long-term Delphi accuracy. Technological Forecasting and Social Change, 78, 1705-1711

Pincombe, B and Pincombe, A (2010). Scoping a Flexible Deployment Framework using Adversarial Scenario Analysis, International Journal of Intelligent Defence Support Systems, 3(3/4), 225-262.

Pincombe, B, Blunden, S, Pincombe, A and Dexter, P (2013), Ascertaining a hierarchy of dimensions from time-poor experts: Linking tactical vignettes to strategic scenarios, Technological Forecasting \& Social Change, 80, 584-598.

Scholz, JB and Reid, DJ, So, where are all the robots? A rationale for strategic research, DSTO technical report in publication.

Williams, PB and Bowden, FDJ, Dynamic morphological exploration, in Gaertner, P., Bowden, F., Piantadosi, J. and Mobbs, K. (eds) 22nd National Conference of the Australian Society for Operations Research (ASOR 2013). The Australian Society for Operations Research, Adelaide, December 2013, pp. 232-238. www.asor.org.au/conferences/asor2013/J8/williams.pdf.

Wright, G and Cairns, G (2011), Scenario Thinking: Practical Approaches to the Future. Houndmills, Hampshire, UK: Palgrave Macmillan. 